\title{
Ultrasound-Guided Percutaneous Ethanol-Paclitaxel Combined Therapy for Rabbit VX2 Liver Tumors
}

\author{
Li Chen' \\ Zhi-xing Liu' \\ Qiu-chen $\mathrm{Bi}^{2}$ \\ Jun Zhao ${ }^{3}$ \\ Qing-rong Liang ${ }^{2}$ \\ Qun Tang ${ }^{2-4}$
}

'Department of Ultrasonic Radiology, The First Affiliated Hospital of Nanchang University, Nanchang, People's Republic of China; ${ }^{2}$ Institute for Advanced Study, Nanchang University, Nanchang, People's Republic of China; ${ }^{3}$ Jiangxi Provincial Key Laboratory of Preventive Medicine, School of Public Health, Nanchang University, Nanchang, People's Republic of China; ${ }^{4}$ Jiangxi Province Key Laboratory of Laboratory Animal, Nanchang, People's Republic of China
Correspondence: Qun Tang Jiangxi Provincial Key Laboratory of Preventive Medicine, School of Public Health, Nanchang University, Xuefu Avenue No. 999, Honggutan District, Nanchang, 33003I, People's Republic of China

Tel/Fax +86 79I-83969963

Email tangqun@ncu.edu.cn
Background: It is difficult to achieve whole tumor ablation using percutaneous ethanol ablation therapy (PEAT) due to the limited diffusion of ethanol.

Purpose: To determine whether chemotherapy can be an adjuvant therapy to benefit PEAT, we investigated ultrasound-guided percutaneous ethanol-paclitaxel combined therapy (PEPCT) of VX2 carcinoma, a rabbit liver cancer model.

Materials and Methods: A six-arm study was designed to quantify the correlation between paclitaxel (PTX) dose and tumor necrosis or cell proliferation, including sham group (2 mL saline, $n=6)$, incremented dose of PTX $(0,12.5,25,37.5 \mathrm{mg})$ in $2.0 \mathrm{~mL}$ ethanol $(\mathrm{n}=6)$ and a conventional PEAT group $(n=6)$ as comparison. The test was followed by contrastenhanced ultrasonic (CEUS) before 7-day sacrifice, tumor harvest, and sectioning. Tumor necrosis ratio was radiologically and histologically quantified; modified proliferation index ( $m$-PI) was proposed to quantify the PTX's pharmacological effects. A linear regression model was set to correlate the PTX dose with tumor necrosis ratio or cell proliferation index. The difference of radiological, histological necrosis ratio (HNR) and modified PI in six groups was analyzed via Kruskal-Wallis $H$-test, Welch analysis of variance and one-way ANOVA.

Results: Incremental increases of PTX $(0,12.5,25,37.5 \mathrm{mg})$ correlated with greater fraction of tumor necrosis $\left(\mathrm{R}^{2}=0.946, \mathrm{P}<0.001\right.$ for radiological necrosis ratio [RNR], $\mathrm{R}^{2}=0.843$, $\mathrm{P}<0.001$ forHNR), indicating that one week after procedure PTX's anti-proliferation and ethanol's dehydration co-induced severe tumor necrosis. Correlation analysis further testified a significant association between PTX dose and $m$-PI $\left(\mathrm{R}^{2}=0.860, \mathrm{P}<0.001\right)$.

Conclusion: These results suggest a clear role for PTX-induced cytotoxicity and support the use of chemotherapeutic drugs in ablation therapy.

Keywords: percutaneous ethanol ablation therapy, PEAT, paclitaxel, PTX, new indication, combined therapy, VX2 liver cancer model

\section{Introduction}

Percutaneous ethanol ablation therapy (PEAT) is effective for patients with small hepatocellular carcinoma (HCC) lesions and poor hepatic functional reserve. PEAT involves the ultrasound-guided intratumoral injection of absolute ethanol into HCC, resulting in cellular dehydration, coagulation necrosis, and vascular thrombosis within the treated tumor. ${ }^{1}$ The patient outcome for PEAT is comparable to those who undergo surgical resection. ${ }^{2}$ However, the rate of local residual recurrence after PEAT is reported to range from $14-44 \%$, owing to viable tumor residue after incomplete ablation. ${ }^{3}$ The 
incomplete ablation is attributed to ethanol's limited spatial diffusion in that viable tumor tissues along the edge of the tumor or in the portions isolated by septa remained intact. ${ }^{4}$ Clinically ethanol is injected into different portions of the lesion with experiential dose and multi-session, which causes tumor necrosis very fast. As a result, massive necrotic components and refluxed ethanol diffuse into vital structures, resulting in abdominal pain, fever, or neurologic deficits. ${ }^{5,6}$ PEAT has evident inferiority to other ablation techniques in terms of overall survival, complications, tumor control in the larger lesion, ease operation in fewer treatment sessions. ${ }^{7,8}$ However, some patients are unsuitable for thermo-ablation due to thermal effect related contraindications, ${ }^{9}$ and for those patients chemo-ablation might be better if the issue of incomplete ablation can be addressed.

Some anticancer drugs (e.g., Doxorubicin, Irinotecan) have been shown to enhance interventional therapy of HCC. As a first-line antineoplastic drug, paclitaxel (PTX) stabilizes microtubule polymerization, thereby arresting cancer cell division. ${ }^{10-12}$ PTX has been clinically approved to treat ovarian, breast, endometrial, and lung cancers. ${ }^{13}$ However, the indication of PTX does not include liver cancers. Two clinical trials on PTX treatment of HCC have been reported: in the 1990s Strumberg et al and Chao et al each concluded the similarly negative results at regular dosing strategies, respectively, ${ }^{14,15}$ and there have been no more clinical trial since that time.

PTX is poorly water soluble $(<0.1 \mu \mathrm{g} / \mathrm{mL})$, but formulation with Cremophor EL (CrEL) to improve solubility often causes severe adverse events. By contrast, PTX has solubility as high as $40 \mathrm{mg} / \mathrm{mL}$ in ethanol, yielding a clear, colorless solution potentially suitable for percutaneous delivery to HCC lesions. Ethanol dehydrates and denatures tumor tissue immediately. Simultaneously, the PTX solute is expected to gradually arrest the proliferation of the tumor cells, particularly the viable residue surviving after ethanol's incomplete ablation. Therefore, we hypothesize that percutaneous ethanol-paclitaxel combined therapy (PEPCT) has a better therapeutic effect than conventional PEAT, where ethanol is not only a chemo-ablation agent, but also a solvent and carrier of PTX. PEPCT might help to clear viable tumor residue via the synergistic ablation-chemotherapy, reducing ethanol dose as well as procedure sessions.

Cell death produced by PEPCT is expected to provide additive results of both ethanol-induced coagulation necrosis and PTX-induced cytotoxicity, and the latter eventually causes superposed necrosis. To determine whether chemotherapy can provide adjuvant benefit PEAT, we aimed to correlate PTX delivery and tumor necrosis after PEPCT in an animal liver cancer model — with the hypothesis that ethanol dissolved with more chemotherapeutic agent would produce more necrosis in ablation-treated tumors - and to investigate the basic biological processes that underlie cell death after PEPCT.

\section{Materials and Methods VX2 Rabbit Liver Cancer Model for PEPCT}

This study was approved by our institutional ethics committee (Ethics Committee, Nanchang University, Approved Number: 20-542). All experiments were conducted in conformity with institutional guidelines for the care and use of laboratory animals in Nanchang University, and the National Institutes of Health Guide for Care and Use of Laboratory Animals. Rabbit VX2 liver cancer model was processed under the guidance of ultrasound imaging, according to the standard protocol described in the reference. ${ }^{16}$ Briefly, frozen rabbit VX2 tumor samples were defrosted and injected into the hind limb muscle of donor New Zealand white rabbits $(3.0 \pm 0.5 \mathrm{~kg})$ for incubation. Approximately 2-3 weeks after implantation, the donor rabbits were anesthetized, the hind-limb tumors were excised and transected into several 1-2 $\mathrm{mm}$ pieces of the tumor and placed in $4^{\circ} \mathrm{C}$ Hanks solution for liver implantation. All the recipient animals were anesthetized by intraperitoneal injection of $3 \%$ pentobarbital solution $(1 \mathrm{~mL} / \mathrm{kg})$. The abdomen was shaved and prepared using Povidone iodine. A 9.0-MHz linear transducer at a low mechanical index (0.10-0.40) (LOGIQ E9 US scanner; GE Healthcare, Pittsburgh, PA) was used for ultrasound-guided VX2 tumor implantation. The tumor was implanted in liver tissue at least $2 \mathrm{~cm}$ in thickness, which was not adjacent to any large vessels. During implantation, an 18-gauge Biopsy needles (inradium $1.2 \mathrm{~mm}$, Mantova, Italy) consisting of a cannula and a core was employed. Two to three fragments of tumor tissue were placed into the lumen of the cannula, followed by one small piece of gelatin foam. Under ultrasound guidance, the needle was inserted into the left lobe of the liver. The tumor fragment was unloaded and gelatin foam were sequentially patched to prevent its leakage from the liver. The puncture needle was removed after implantation and then the animals were aroused and returned to their cages. The size of tumor lesions was daily monitored by B mode ultrasonic imaging from the 10th day after implantation. Provided that the 
implanted tumor grew up to 1.5 to $2 \mathrm{~cm}$ the rabbit was qualified for the procedure.

\section{Ablation Procedures}

Animals were pre-anesthetized (35-50 mg/kg ketamine, $5 \mathrm{mg} / \mathrm{kg}$ xylazine, and $0.01-0.02 \mathrm{mg} / \mathrm{kg}$ glycopyrrolate) intramuscularly, and placed in a supine position. After iodine disinfection, 23-gauge Biopsy needles (in-radium $0.2 \mathrm{~mm}$, Mantova, Italy) punctured the VX2 tumor under ultrasound guidance (LOGIQ E9 US scanner; GE Healthcare, Pittsburgh, PA) with a $9.0-\mathrm{MHz}$ linear transducer at a low mechanical index $(0.10-0.40)$ Absolute ethanol (Pharmaceutical Secondary Standard, Sigma), or ethanol mixed with a specific dose of PTX (United States Pharmacopeia, Sigma) was slowly injected $(0.1 \mathrm{~mL} / \mathrm{S})$. To maximize spatial uniformity of drug distribution. We divided the tumor into three parts along the largest diameter, and half the volume of the injection was released into the bottom part ( $1 / 3$ point), then the other half into the top part ( $2 / 3$ point) (Figure 1A). All the procedures were performed by a single operator $(\mathrm{CL})$ to minimize interoperator variability.

A six-arm study was designed to quantify the correlation between PTX and tumor necrosis (Figure 1B). In the sham group, $2 \mathrm{~mL}$ saline was prepared for percutaneous injection $(\mathrm{n}=6)$. Incremented dose of PTX $(0,12.5,25,37.5 \mathrm{mg}$ in $2.0 \mathrm{~mL}$ ethanol, $\mathrm{n}=6$ ) in group 2, 4, 5 and 6 was used for correlation study. As a comparison Group 3 was set as conventional PEA therapy $(n=6)$, where the total volume $(\mathrm{mL})$ of ethanol to be injected was determined by clinical dose, as calculated by an empirical formula: $4 / 3 \pi(r+0.5)^{3}$, where $\mathrm{r}$ is the radius of the tumor $(\mathrm{cm}) .{ }^{17}$ On the 7 th day after ablation, all the rabbits were tested by contrast-enhanced ultrasonic (CEUS) and then killed for pathologic analysis.

\section{CEUS Imaging}

Radiological necrosis ratio (RNR) was measured with CEUS

7 days after ultrasound intervention therapy on the same scanner. Rabbits were anesthetized and CEUS was recorded following bolus injection of $0.5 \mathrm{~mL}$ SonoVue solution into the ear margin vein, followed by a saline flush $(5 \mathrm{~mL} 0.9 \%$ $\mathrm{NaCl}$ ). Serial dynamic images were acquired until the contrast agent diminished, and RNR was quantitatively calculated as (whole tumor area - viable residue area)/whole tumor area $* 100 \%$. Both areas were automatically measured on the images acquired during the arterial phase. Note that RNR before therapy is almost undetectable in all groups, it is reasonable as the tumor size is less than $2 \mathrm{~cm}$. All the data were obtained by a single investigators (ZXL).

\section{Pathologic Process of Tumor Samples}

All animals were sacrificed by means of intravenous pentobarbital (Veterinary Laboratories, Lenexa, Kan) overdose immediately after CEUS. The whole VX2 tumors were resected and fixed in formalin. Formalin-fixed VX2 tumors were embedded in paraffin, sectioned at 5-mm thickness through the center of the tumor at the largest diameter, and mounted onto glass slides (Star Frost Plus; Mercedes Medical, Sarasota, Fla). For histologic examination and immunohistochemical (IHC) staining, two adjacent slices at the maximum cross-section were obtained with a thickness of $5 \mu \mathrm{m}$. One slice was stained by standard hematoxylin-eosin staining protocol, and the neighbored slice stained by $\mathrm{Ki}-67$ antibodies to assess tumor cell proliferation. Histological necrosis ratio (HNR) is a relatively precise standard for validated therapeutic response schemes associated with survival outcomes after local-regional therapy for HCC. ${ }^{18}$ It was estimated as follows: necrotic area/whole tumor area $\times 100 \%$, autoread by the software "image-pro plus 6.0". All the data were obtained by a single investigators (JZ)

We proposed modified proliferation index $(m-\mathrm{PI})$ to faithfully describe the VX2 cell's proliferation ability after survival from ethanol ablation $(m-\mathrm{PI}=$ number of KI-67 positive cells/the whole viable cells $* 100 \%$ ). In detail, the whole viable cells from $H \& E$ slice were counted at a certain viewpoint (e.g., 10 viable cells at $40 \times$ magnification), and then,
A

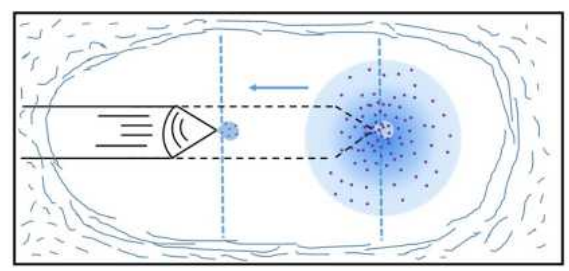

B

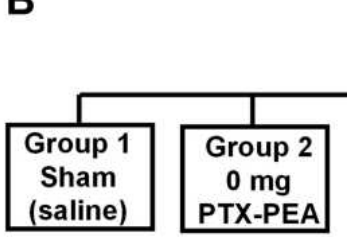

6-arm study

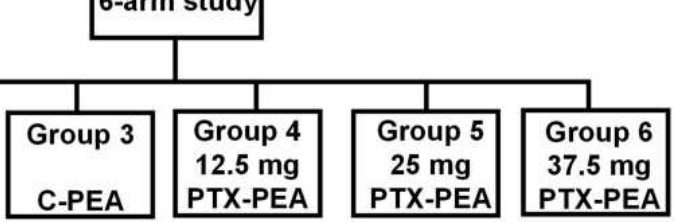

Figure I (A) Scheme of the PEPCT technique: The infusion needle is punctured percutaneously into the lesion under the guidance of ultrasound imaging. The half volume of ethanol is injected into I/3 point, the other half at 2/3 point. (B) Organizational chart of the study design, consisting of four treatment arms with the PTX varying in the dose in ethanol solvent and conventional PEA (C-PEA). 
from the same viewpoint in the neighboring slice the KI-67 positive cells were identified from those viable cells (e.g., among the 10 viable cells, only 8 cells are positive, then $m$-PI is counted as $80 \%$ ). For each slice we randomly selected three areas and averaged the values as each tumor's $m$-PI value. All the data were obtained by a single investigators (QRL)

\section{Statistical Analysis}

All the data are expressed as Mean \pm SD. Statistical analyses were performed using SPSS 22.0 (SPSS Inc., Chicago, IL, USA) and GraphPad Prism 6.01 (GraphPad Software, USA). The relationship between PTX dose and radiological/histological necrosis or proliferation index was assessed by using least-squares linear regression with the calculation of coefficients of determination $\left(\mathrm{R}^{2}\right)$, all the data testing meets the assumptions of linear regression. A Welch analysis of variance was performed to compare the differences of HNR among all the groups and Games-Howell test was performed to make pairwise comparisons between independent groups. Kruskal-Wallis $H$-test and Bonferroni correction method were performed to compare the differences of RNR among all the groups and to make pairwise comparisons between independent groups, respectively. One-way ANOVA and Tukey's test were performed to compare the differences of $m$-PI among all the groups and to make pairwise comparisons between independent groups, respectively. $\mathrm{P}$ values $<0.05$ were considered to indicate a statistically significant difference, and $\mathrm{P}$ values $<0.1$ represented a statistical trend.

\section{Results}

Procedures and Typical Photograph of the Resected Specimen

A total of 36 New Zealand white rabbits was used for therapy. All the procedures (100\%) were technically successful after repeated practice for three months. No procedure-related complications or mortality were encountered.
Photograph of the tumor tissue are shown in Figure 2. In contrast to pure ethanol, PTX solute in ethanol caused more necrosis, and in cases of the high dose group no viable residue was found. Photographs are presented for all tumors in the Appendix (Figure S1).

\section{Radiological Tumor Necrosis and Histological Tumor Necrosis}

Tumor radiological necrosis was found to be different between groups (Figure 3A). Subset analysis indicated that the increase of RNR treated by C-PEI was highly significant compared with the sham group $(\mathrm{P}<0.001)$, moderately significant compared with the $2 \mathrm{~mL}$ ethanol group $(\mathrm{P}=0.048)$, but not significant compared with the low PTX dose group $(\mathrm{P}=0.307)$. Therefore, administration of high dose of ethanol was relatively beneficial, since the RNR value was unsatisfactorily $(52.75 \pm 8.92 \%)$ at the clinically-used dose. Meanwhile, relative to $\mathrm{C}-\mathrm{PEI}$, the RNR for the mediumdose group was about $50 \%$ greater $(77.97 \pm 8.17 \%, \mathrm{P}=0.020)$. For the high dose group, the difference is statistically significant, since for all the tumors only very weak signal of dynamic enhancement was detected $(95.00 \pm 3.58 \%$, $\mathrm{P}<0.001$ ). HNR was less than RNR (Figure 3B), but the statistical differences and trends were similar. Necrosis ratio in C-PEI is significantly higher than that in sham $(\mathrm{P}=0.003)$, comparable with $2 \mathrm{~mL}$ PEI $(\mathrm{P}=0.380)$, low $(\mathrm{P}=0.989)$ and medium dose group $(\mathrm{P}=0.291)$, but remarkably lower than the high dose group $(\mathrm{P}<0.001)$. Note that clinically the radiological necrosis of HCC is generally higher than that of histological outcome, as we did. An individual archive including the arterial phase images, H\&E scanning images, RTR and HTR values are shown in Appendix (Figure S2 and S3).

\section{Relationship Between PTX Dose and Tumor Necrosis}

Linear regression showed a strong positive relationship between administered PTX dose and RNR $\left(\mathrm{R}^{2}=0.946\right.$;

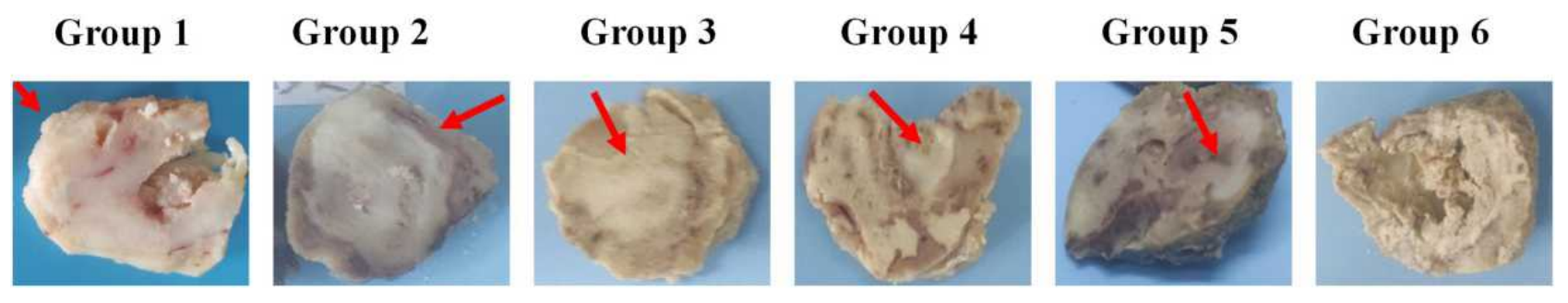

Figure 2 A typical cut surface photograph of the resected specimen of VX2 tumors. Generally, the white area is considered as viable tumor residue. In the saline group (group I), the whole tumor looks highly viable. Ablation or synergistic ablation-chemotherapy treatment caused necrosis at different levels, Extremely, at the highest dose of PTX (group 6), the VX2 tumor is almost completely necrotic. 

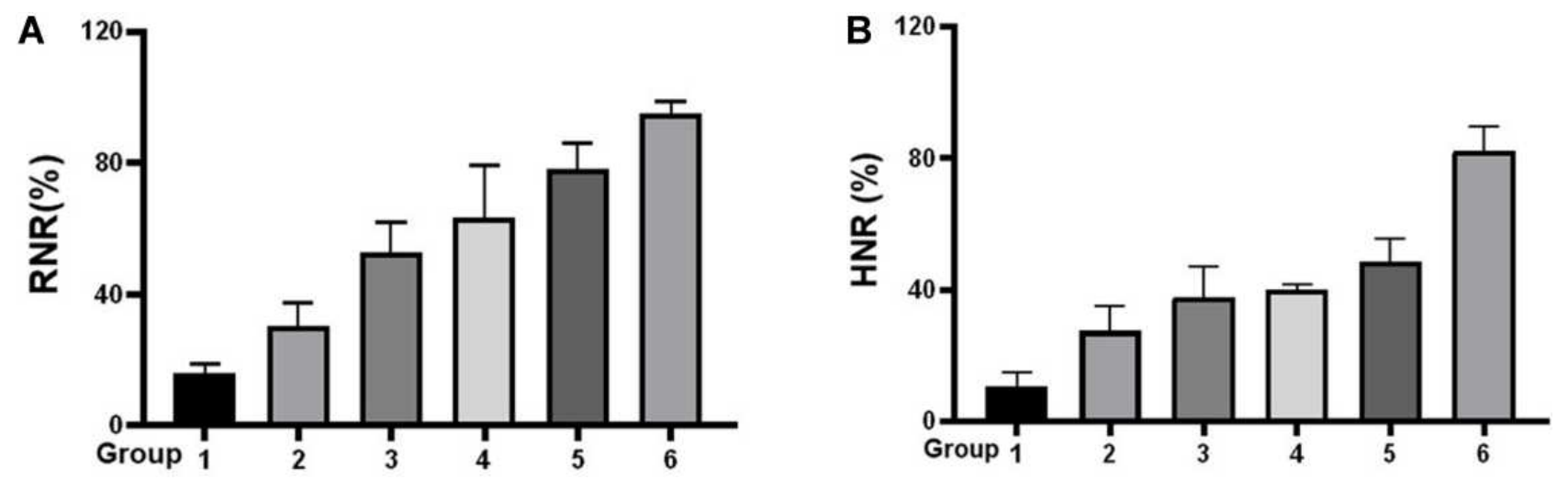

Figure 3 (A) The RNR values in all six groups were determined by the enhanced area recorded at the arterial phase of CEUS measured at one week after the procedure. (B) HNR values in all six groups were determined by automatically reading the H\&E slices.

Figure 4A). The dose of the drug has a statistically significant effect on the necrosis ratio $(\mathrm{F}(1,16)=154.389, \mathrm{P}<0.001)$, the dose of the drug attribute to $90.6 \%$ of the variation of the necrosis ratio. HNR showed a similar trend $\left(\left(\mathrm{R}^{2}=0.843\right.\right.$; Figure 4B), $\mathrm{P}<0.001)$. Correlation analysis demonstrated a significant association between PTX dose and tumor necrosis, indicating that PTX exerted cytotoxicity on the VX2 cells.

\section{Modified Proliferation Index $(m-\mathrm{Pl})$ for Quantitative Cytotoxicity of PTX}

The Ki-67 protein is expressed in all phases of the cell cycle except G0 and serves as a good marker for proliferation. High Ki-67 proliferative index, the fraction of tumor cell nuclei above a defined threshold being positive for Ki-67, has been reported to correlate with prognosis of patients with various cancers. The $\mathrm{Ki}-67$ proliferation index is normally assessed by point counting a number of cells and is reported as percentage of positive cells. In this study we utilized $m$-PI to define the PTX's anti-proliferation on those VX2 cells surviving ethanol ablation, which more precisely describes the extent to which viable cells have lost proliferation ability. As shown in Figure $5 m$-PI has no significant difference between three non-PTX groups $(\mathrm{P}=0.642)$, indicating ethanol ablation has negligible anti-proliferation effect on VX2 cells. As PTX dose increases, fewer viable cells are Ki-67 positive as correlation analysis indicated the negative relation between administered PTX dose and $m$-PI $\left(\mathrm{R}^{2}=0.860, \mathrm{P}<0.001\right)$, it means that more and more percentages of viable cells cannot divide anymore although they survive the synergistic therapy, at high dose group only $6.23 \pm 1.98 \%$ viable cells are positive, much lower than that in the C-PEI group $(60.48 \pm 5.00 \%, \mathrm{P}<0.001)$.
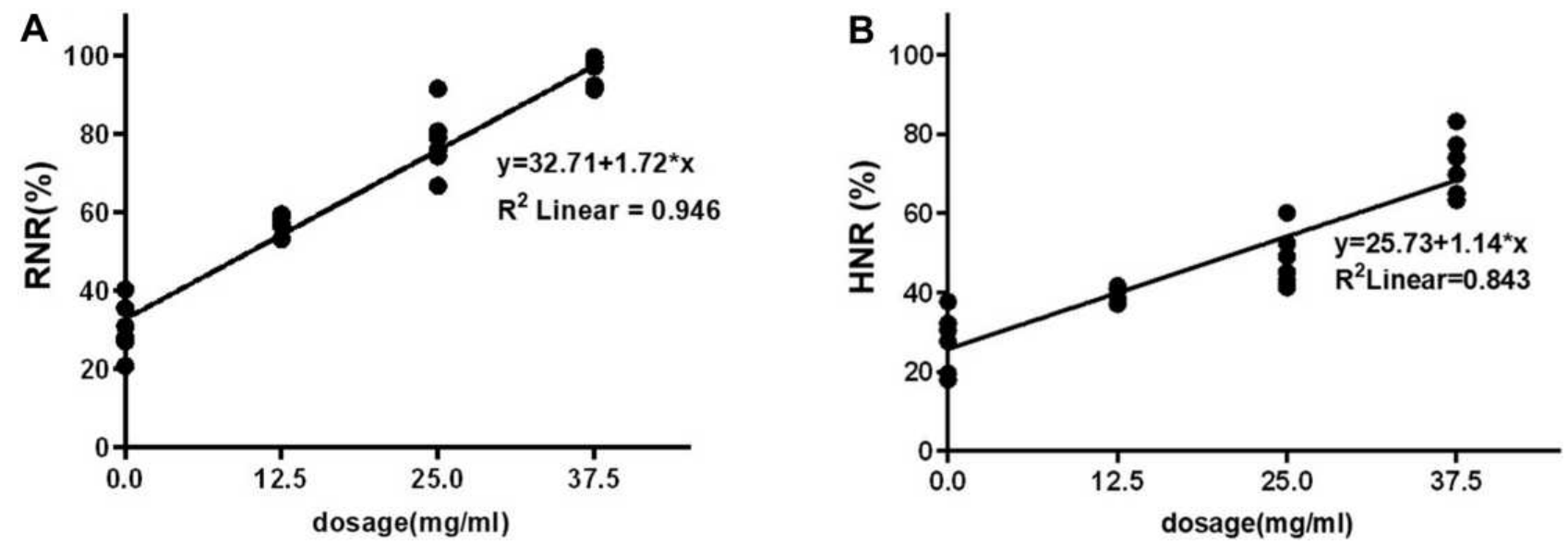

Figure 4 Scatterplot of PTX dose versus percentage tumor necrosis ratio with a best-fit regression line shows a strong linear relationship between these parameters. The slope indicated a dose dependent response: the necrosis ratio increases radiologically (A) by I.721\%/mg (95\% Cl:I.535-I.907) and histologically (B) by I.I4\%/mg ( $95 \%$ Cl:0.92-1.35), respectively. 


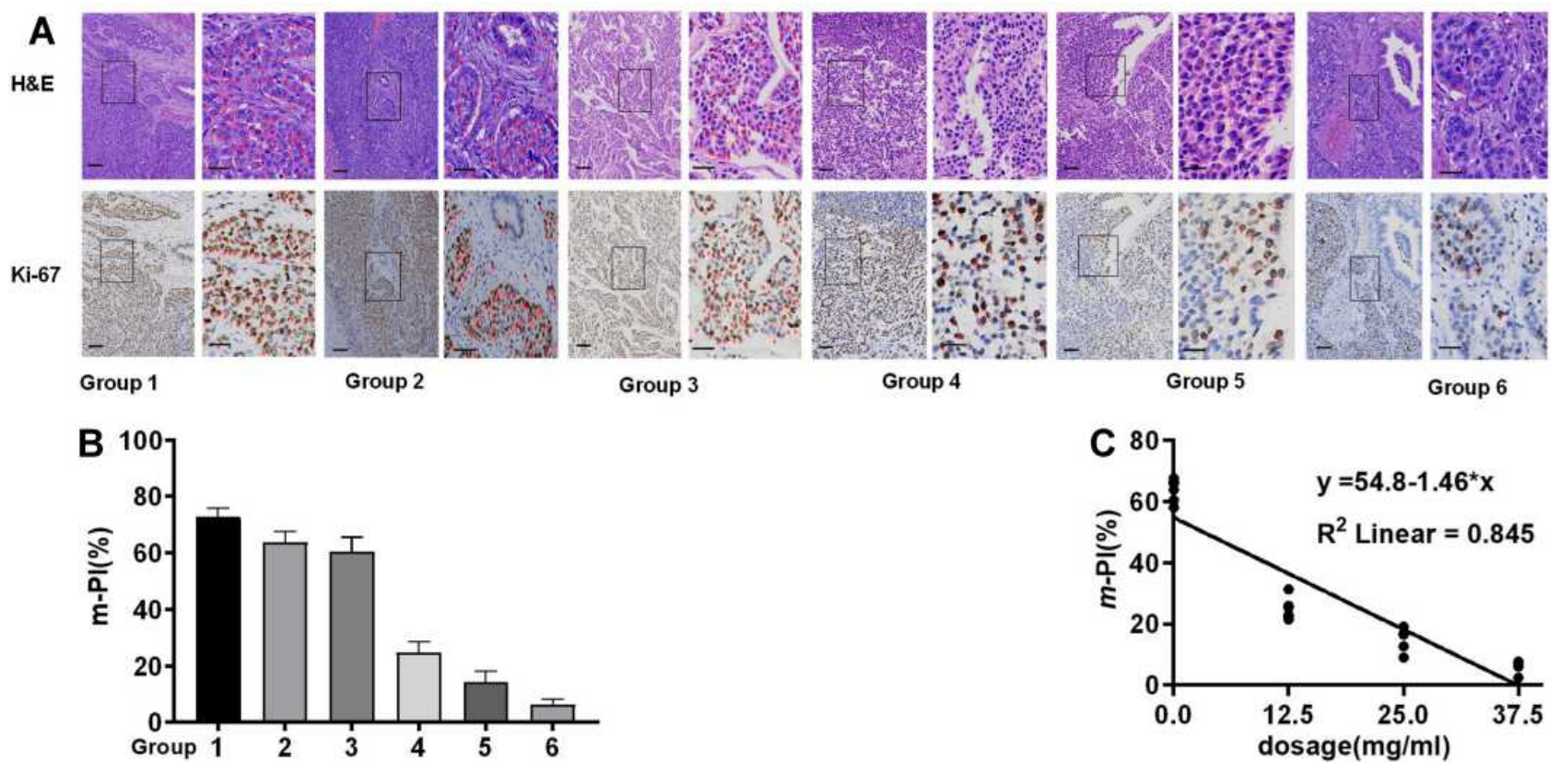

Figure 5 (A) Typical photographs for $m$-PI measured. Red asterisks represent the viable, and well-proliferated (Ki-67 positive) cells. Scale bar at the low magnification (I0x): $100 \mu \mathrm{m}$; at the high magnification (40×): $30 \mu \mathrm{m}$. (B) Individual $m$-PI values. (C) Scatterplot of PTX dose versus $m$-PI.

\section{Discussion}

We have showed that addition of PTX enhanced the conventional PEAT at low dose of ethanol after one session procedure. Both radiological and pathological necrosis ratios and modified-proliferation index correlated with the dose of PTX. This combined therapy illustrated a synergistic effect between ablation and chemotherapy, indicating PEPCT could be an alternative therapeutic strategy for the interventional treatment of liver cancer.

Reflux of ethanol determines its dehydrating and denaturing effect. ${ }^{19}$ Ethanol's spatial diffusion is driven by the concentration gradient and therefore the local concentration diminishes towards the rim of the tumor and may be insufficient to local recurrence and metastasis. ${ }^{20}$ PTX inhibits the proliferation of those cells survived ethanol ablation. In vitro tests described in the Appendix showed that incubation with $5 \%$ ethanol killed about half of the VX2 or SMMC-7721 cells (purchased from the Shanghai Institutes for Biological Sciences), but once the ethanol was removed, the cell population rebounded to the pre-stimulation level within 72 hours. In contrast, PTX can inhibit the rebound on a dose-dependent manner (Figure S4). This synergistic effect is consistent with our observation in vivo. Particularly, comparison of $m$-PI directly evidenced this mechanism. The PTX might have a close spatial distribution with ethanol due to the enormous divergence of solubility of PTX in ethanol and water.
Therefore, even though only small amount of ethanol diffuses close to the rim, co-delivered PTX can still arrest the viable tumor cells' proliferation. Although those cells seems "viable", due to lack of proliferation ability they have low chance to invade or metastasize, thereby reducing the risk of recurrence. The long-term effect is still under investigation.

On the other hand, we found a new indication for PTX. As an anti-proliferative agent, PTX has shown cytotoxic activity in human HCC cell lines. ${ }^{21,22}$ The reason for its clinical failure is still unexplored. This combination of ethanol ablation and PTX has been clinically applied to treat pancreatic cysts. Hyoung-Chul Oh proposed that PTX is highly hydrophobic and viscous; therefore, it can exert its effect longer when instilled within the cyst cavity with a low risk of leakage through a puncture site and causing complications. ${ }^{23}$ EUS-guided cyst ablation using PTX have reported numerically better response rates although they reminded that the diffused PTX injured epithelial cells and provide additional inhibitory effects of apoptosis on epithelial lining.This positive results reminds us that PTX might also have a similar enhancement effect on ethanol ablation of liver cancer. ${ }^{24}$ In this preclinical PEPCT assessment, PTX exerts its anticancer effect via US-guided intratumoral administration, where PTX more directly interact with liver cancer cells. PTX freely accessed to tumor cells and exerted a quasi-in vitro anticancer effect. As reported, intratumoral delivery of PTX showed much higher efficacy than other 
modes of administration on an orthotopic prostate cancer model, ${ }^{25}$ additionally transcatheter arterial chemoembolization with the PTX-lipiodol solution has also proved to activate against VX2 liver tumors. ${ }^{26}$ Our results suggest that route of administration may determine PTX effectiveness.

Finally, thermo-ablation techniques (radiofrequency, microwave, laser) is predominantly accepted for the treatment of liver cancer, and chemo-ablation is gradually being phased out. ${ }^{27}$ However, contraindication for thermoablation must be concerned, for example, gallbladder or gastrointestinal perforation, angiorrhagia might occur depending on the lesion's position, ${ }^{9}$ for example, the blood supply in the liver is very rich, and some tumor lesions are very close to the blood vessel, therefore the risk of thermo-ablation procedure will be quite higher, although some tricky solution has been tried to prevent the local overheating. For those patients PEPCT might be an ideal alternative treatment strategy according to the advantage of PEAT over percutaneous radiofrequency ablation. ${ }^{28}$ Moreover, the addition of PTX into ethanol ablation remarkably alleviates the dose of ethanol, a big safe issue of PEAT. $^{29}$ Finally, ethanol ablation is still competitive for the undeveloped area and countries considering the procedure's cost.

This study still does have some limitations. The correlation between spatiotemporal evolution of cellular behavior and PTX distribution has not been investigated. Also, systemic toxicity remains unknown, particularly the potential risks under the high dose of PTX. Additionally, the longtermed local recurrence of PEPCT has not been evaluated to compare with PEAT or radiofrequency ablation. Finally, the study examined a relatively small size of animals over a relatively short term, and further studies that involve larger samples and longer follow-ups are warranted.

In conclusion, noting the pharmacological properties of PTX, a solution in ethanol was developed to treat the HCC model under ultrasound guidance. The positive results remind us that PPECT might have a similar or superior clinical outcome to other ablation techniques. Clinical trials are under development.

\section{Acknowledgments}

This work was funded by the National Natural Science Foundation of China (grant numbers 21661022, 82060335), Natural Science Foundation of Jiangxi Province (20192BCD40003). The authors acknowledge histology technologist from Department of Pathology in the first affiliated hospital to Nanchang University, for assistance with digitization of histological slides used for analysis. Dr. Qun Tang thanks Prof. Ralph Mason (University of Texas Southwestern Medical Center) for a critical reading of the manuscript.

\section{Disclosure}

The authors reported no conflicts of interest for this work.

\section{References}

1. Livraghi T, Festi D, Monti F, Salmi A, Vettori C. US-guided percutaneous alcohol injection of small hepatic and abdominal tumors. Radiology. 1986;161(2):309-312. doi:10.1148/radiology.161.2.3020612

2. Castells A, Bruix J, Bru C, et al. Treatment of small hepatocellular carcinoma in cirrhotic patients: a Cohort Study comparing surgical resection and percutaneous ethanol injection. Hepatology. 1993;18 (5):1121-1126.

3. Ishii $\mathrm{H}$, Okada $\mathrm{S}$, Nose $\mathrm{H}$, et al. Local recurrence of hepatocellular carcinoma after percutaneous ethanol injection. Cancer. 1996;77 (9):1792-1796. doi:10.1002/(SICI)1097-0142(19960501)77:9<1792:: AID-CNCR6 $>3.0 . \mathrm{CO} ; 2-\mathrm{E}$

4. Shiina S, Tagawa K, Unuma T, et al. Percutaneous ethanol injection therapy for hepatocellular carcinoma. A Histopathologic Study. Cancer. 1991;68(7):1524-1530. doi:10.1002/1097-0142(19911001) 68:7<1524::AID-CNCR2820680711>3.0.CO;2-O

5. Zheng SG, Xu HX, Lu MD, et al. Role of contrast-enhanced ultrasound in follow-up assessment after ablation for hepatocellular carcinoma. World J Gastroenterol. 2013;19(6):855-865. doi:10.3748/wjg.v19. i6.855

6. Shiina S, Tateishi R, Imamura M, et al. Percutaneous ethanol injection for hepatocellular carcinoma: 20-year outcome and prognostic factors. Liver Int. 2012;32(9):1434-1442. doi:10.1111/j.14783231.2012.02838.x

7. Bouza C, Lopez-Cuadrado T, Alcazar R, Saz-Parkinson Z, Amate JM. Meta-analysis of percutaneous radiofrequency ablation versus ethanol injection in hepatocellular carcinoma. BMC Gastroenterol. 2009;9(1):31. doi:10.1186/1471-230X-9-31

8. Orlando A, Leandro G, Olivo M, Andriulli A, Cottone M. Radiofrequency thermal ablation vs. percutaneous ethanol injection for small hepatocellular carcinoma in cirrhosis: meta-analysis of randomized controlled trials. Am J Gastroenterol. 2009;104 (2):514-524. doi:10.1038/ajg.2008.80

9. Bilchik AJ, Wood TF, Allegra DP. Radiofrequency ablation of unresectable hepatic malignancies: lessons learned. Oncologist. 2001;6 (1):24-33. doi:10.1634/theoncologist.6-1-24

10. Wani MC, Taylor HL, Wall ME, Coggon P, McPhail AT. Plant antitumor agents. VI. The isolation and structure of taxol, a novel antileukemic and antitumor agent from Taxus brevifolia. $J$ Am Chem Soc. 1971;93(9):2325-2327. doi:10.1021/ja00738a045

11. Schiff PB, Horwitz SB. Taxol stabilizes microtubules in mouse fibroblast cells. Proc Natl Acad Sci U S A. 1980;77(3):1561-1565. doi:10.1073/pnas.77.3.1561

12. Schiff PB, Fant J, Horwitz SB. Promotion of microtubule assembly in vitro by taxol. Nature. 1979;277(5698):665-667. doi:10.1038/ 277665a0

13. Rowinsky EK, Donehower RC, Wood AJJ. Paclitaxel (taxol). $N$ Engl J Med. 1995;332(15):1004-1014. doi:10.1056/NEJM199504133321507

14. Strumberg D, Erhard J, Harstrick A, et al. Phase I study of a weekly 1 $\mathrm{h}$ infusion of paclitaxel in patients with unresectable hepatocellular carcinoma. Eur J Cancer. 1998;34(8):1290-1292. doi:10.1016/ S0959-8049(98)00054-9 
15. Chao Y, Chan WK, Birkhofer MJ, et al. Phase II and pharmacokinetic study of paclitaxel therapy for unresectable hepatocellular carcinoma patients. Br J Cancer. 1998;78(1):34-39. doi:10.1038/bjc.1998.438

16. Zou X, Liu Q, Zhou X, et al. Ultrasound-guided percutaneous laser and ethanol ablation of rabbit VX2 liver tumors. Acta Radiol. 2013;54(2):181-187. doi:10.1258/ar.2012.110723

17. Shiina S, Tagawa K, Unuma T, et al. Percutaneous ethanol injection therapy of hepatocellular carcinoma: analysis of 77 patients. AJR Am $J$ Roentgenol. 1990;155(6):1221-1226. doi:10.2214/ajr.155.6.217 3384

18. Memon K, Kulik L, Lewandowski RJ, et al. Radiographic response to locoregional therapy in hepatocellular carcinoma predicts patient survival times. Gastroenterology. 2011;141(2):526-535, 535e521522. doi:10.1053/j.gastro.2011.04.054

19. Vehmas T. Reflux of ethanol during experimental liver ethanol injections. Invest Radiol. 1992;27(11):918-921. doi:10.1097/ 00004424-199211000-00008

20. Ohnishi K, Yoshioka H, Ito S, Fujiwara K. Prospective randomized controlled trial comparing percutaneous acetic acid injection and percutaneous ethanol injection for small hepatocellular carcinoma. Hepatology. 1998;27(1):67-72. doi:10.1002/hep.510270112

21. Gagandeep S, Novikoff PM, Ott M, Gupta S. Paclitaxel shows cytotoxic activity in human hepatocellular carcinoma cell lines. Cancer Lett. 1999;136(1):109-118. doi:10.1016/S0304-3835(98) 00388-7

22. Nasongkla N, Nittayacharn P, Rotjanasitthikit A, Pungbangkadee K, Manaspon C. Paclitaxel-loaded polymeric depots as injectable drug delivery system for cancer chemotherapy of hepatocellular carcinoma. Pharm Dev Technol. 2017;22(5):652-658. doi:10.3109/ 10837450.2016 .1163389
23. Oh HC, Seo DW, Lee TY, et al. New treatment for cystic tumors of the pancreas: EUS-guided ethanol lavage with paclitaxel injection. Gastrointest Endosc. 2008;67(4):636-642. doi:10.1016/j. gie.2007.09.038

24. Attila T, Adsay V, Faigel DO. The efficacy and safety of endoscopic ultrasound-guided ablation of pancreatic cysts with alcohol and paclitaxel: a systematic review. Eur J Gastroenterol Hepatol. 2019;31 (1):1-9. doi:10.1097/MEG.0000000000001297

25. Shikanov S, Shikanov A, Gofrit O, Nyska A, Corn B, Domb AJ. Intratumoral delivery of paclitaxel for treatment of orthotopic prostate cancer. J Pharm Sci. 2009;98(3):1005-1014. doi:10.1002/ jps. 21492

26. Yoon CJ, Chung JW, Park JH, et al. Transcatheter arterial chemoembolization with paclitaxel-lipiodol solution in rabbit VX2 liver tumor. Radiology. 2003;229(1):126-131. doi:10.1148/radiol.2291021029

27. Vilana R, Bruix J, Bru C, Ayuso C, Sole M, Rodes J. Tumor size determines the efficacy of percutaneous ethanol injection for the treatment of small hepatocellular carcinoma. Hepatology. 1992;16 (2):353-357. doi:10.1002/hep.1840160212

28. Yu SCH, Hui JWY, Chong CCN, et al. Transarterial ethanol ablation for small hepatocellular carcinoma $(</=3 \mathrm{~cm})$ : a Comparative Study versus radiofrequency ablation. Cardiovasc Intervent Radiol. 2020;43 (5):732-739. doi:10.1007/s00270-020-02426-4

29. Redvanly RD, Chezmar JL, Strauss RM, Galloway JR, Boyer TD, Bernardino ME. Malignant hepatic tumors: safety of high-dose percutaneous ethanol ablation therapy. Radiology. 1993;188(1):283-285. doi:10.1148/radiology.188.1.8390070
Journal of Hepatocellular Carcinoma

\section{Publish your work in this journal}

The Journal of Hepatocellular Carcinoma is an international, peerreviewed, open access journal that offers a platform for the dissemination and study of clinical, translational and basic research findings in this rapidly developing field. Development in areas including, but not limited to, epidemiology, vaccination, hepatitis therapy, pathology and molecular tumor classification and prognostication are all considered for publication. The manuscript management system is completely online and includes a very quick and fair peer-review system, which is all easy to use. Visit http://www.dovepress.com/ testimonials.php to read real quotes from published authors. 